\title{
Simultaneous Measurement of Glass-Transition Temperature and \\ Crystallinity of As-Prepared Polymeric Films from Restitution
}

\section{SUPPORING INFORMATION}

Jinwon Park ${ }^{1}$, Seongsoo Han ${ }^{1}$, Hyeonjung Park ${ }^{1}$, Jaehong Lee ${ }^{1}$, Suchan $\mathrm{Cho}^{2}$, Myungeun $\mathrm{Seo}^{2,3}$, Bumjoon J. Kim ${ }^{1,3}$, and Siyoung Q. Choi ${ }^{1,3 *}$

${ }^{1}$ Department of Chemical and Biomolecular Engineering, Korea Advanced Institute of Science and Technology (KAIST), Daejeon 34141, Korea

${ }^{2}$ Department of Chemistry, Korea Advanced Institute of Science and Technology (KAIST),

Daejeon 34141, Korea

${ }^{3}$ KAIST Institute for the Nanocentury, Korea Advanced Institute of Science and Technology (KAIST), Daejeon 34141, Korea

*corresponding author

Siyoung Q. Choi (sqchoi@kaist.ac.kr) 


\section{NMR results of PLA- $b$-PS}
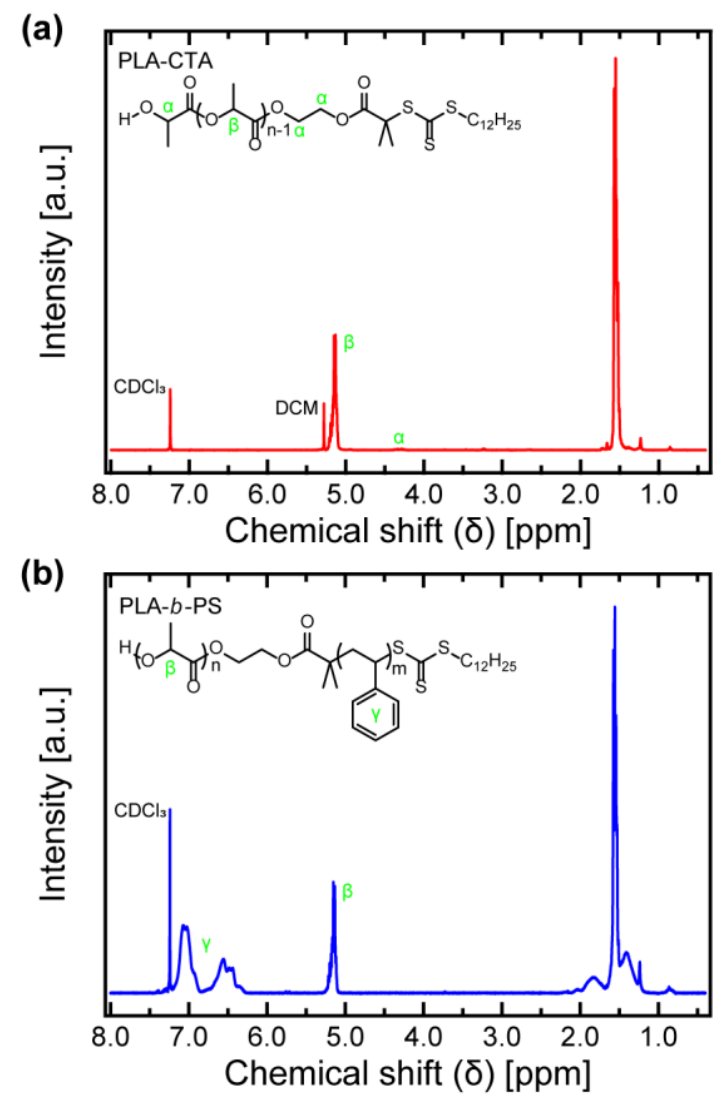

Figure S1: NMR results of (a) PLA-CTA and (b) PLA- $b$-PS with $\mathrm{CDCl}_{3}$ solvent. $M_{\mathrm{n}}$ of PLA-CTA was first calculated by the relative peak integrations at CTA end $\left(\delta\right.$ 4.30) and PLA $\left(\delta\right.$ 5.14). $M_{\mathrm{n}}$ of PLA-b-PS was then calculated according to the relative peak integrations at PLA ( $\delta$ 5.14) and PS ( $\delta 6.52$ and 7.04). Each peak-related protons are marked as $\alpha, \beta$, and $\gamma$. 


\section{NMR results of P3HTs}

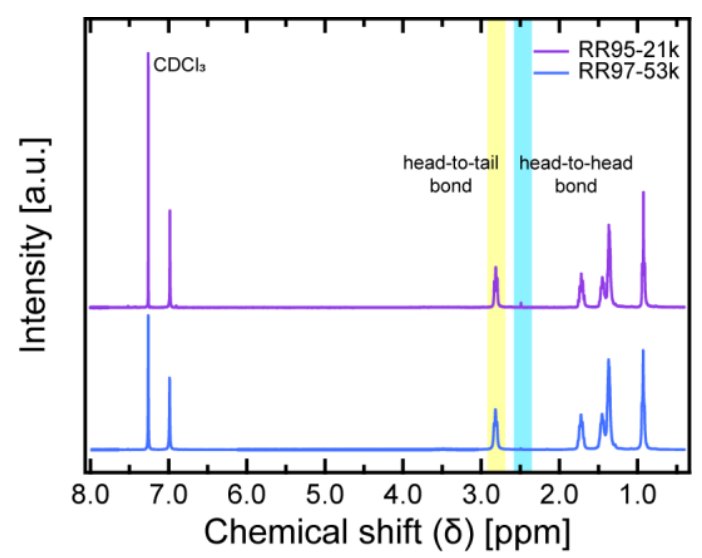

Figure S2: NMR results of P3HTs with low and high $M_{\mathrm{n}}$ (RR95-21k and RR97-53k) with $\mathrm{CDCl}_{3}$ solvent. RR was calculated by the relative peak integrations at head-to-tail bond $\left(\begin{array}{ll}\delta & 2.78\end{array}\right)$ and head-to-head bond $(\delta 2.54)$. 


\section{Ball restitution results at different temperature changing conditions}
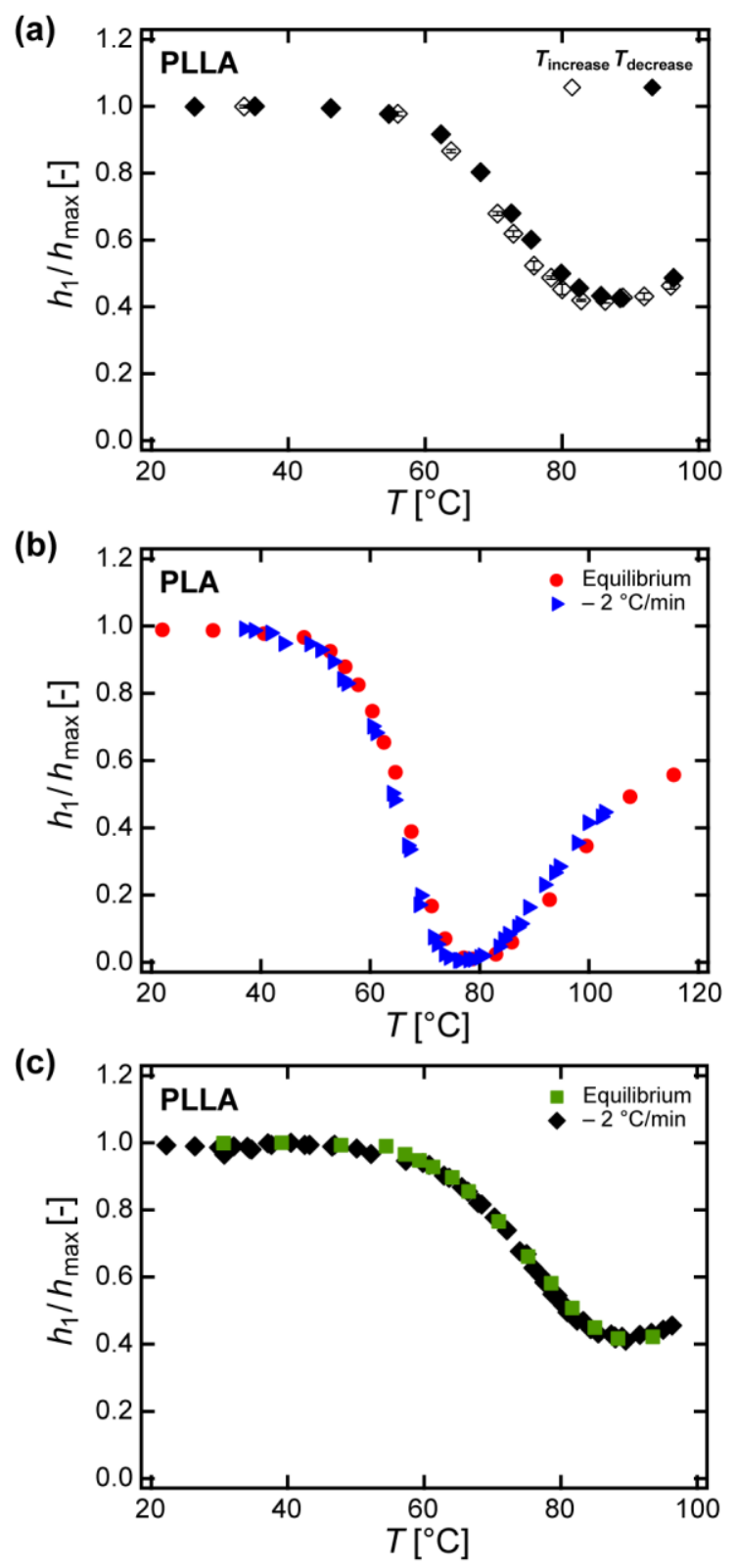

Figure S3: Restitution results on (a) $20 \mathrm{~h}$ annealed PLLA with increasing and decreasing temperatures, (b) PLA with different rates of temperature change, and (c) $26 \mathrm{~h}$ annealed PLLA with different rates of temperature change. For the equilibrium condition experiment, error bars were presented, but they were smaller than the size of the markers. 


\section{Ball restitution results while varying drop height and sizes of the balls}
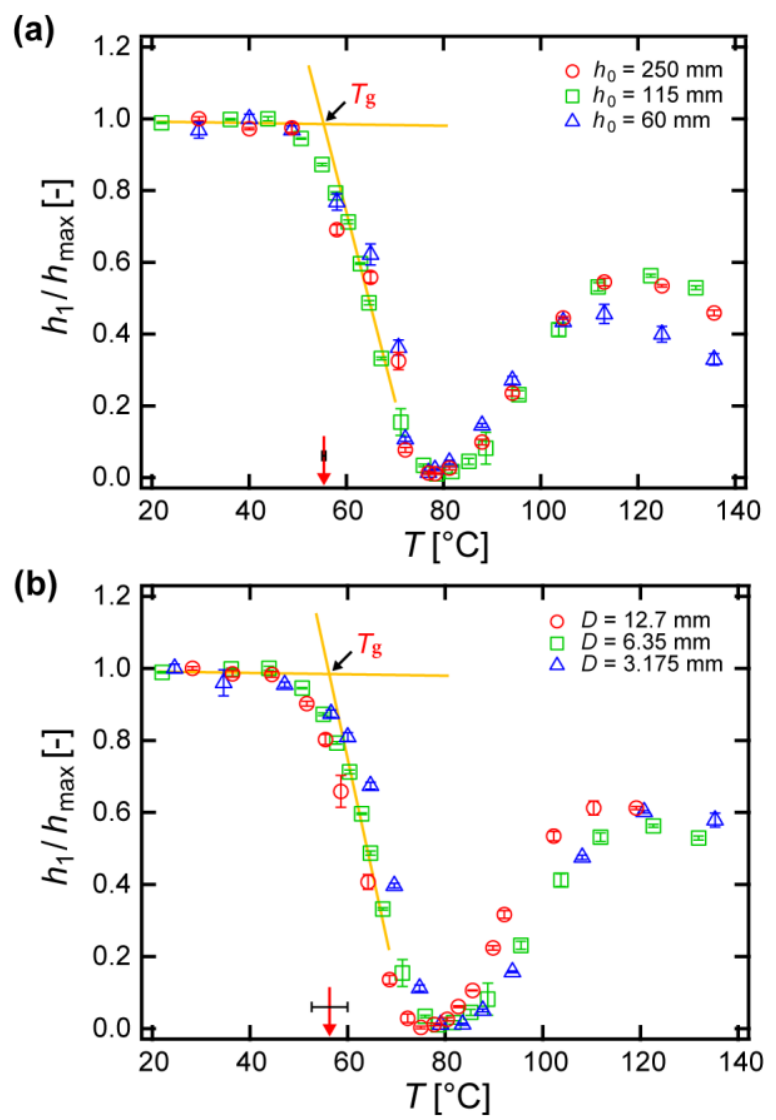

Figure S4: Results of the ball collision experiment on PLA with (a) changing the initial drop height $(250,115$, and 60 $\mathrm{mm})$ and (b) changing the mass and diameter $(12.7 \mathrm{~mm}, 8.50 \mathrm{~g} ; 6.35 \mathrm{~mm}, 1.06 \mathrm{~g}$; and $3.175 \mathrm{~mm}, 0.13 \mathrm{~g})$ of the balls. Two representative yellow guidelines that determine $T_{\mathrm{g}}$ are shown, and the measured $T_{\mathrm{g}}$ with different conditions are marked with red arrows with black error bars at the bottom of each graph. The measured $T_{\mathrm{g}}$ results are (a) $T_{\mathrm{g}}=$ $55.4 \pm 0.7^{\circ} \mathrm{C}$ and (b) $T_{\mathrm{g}}=56.0 \pm 3.7^{\circ} \mathrm{C}$. 


\section{Ball restitution results while varying film thickness}
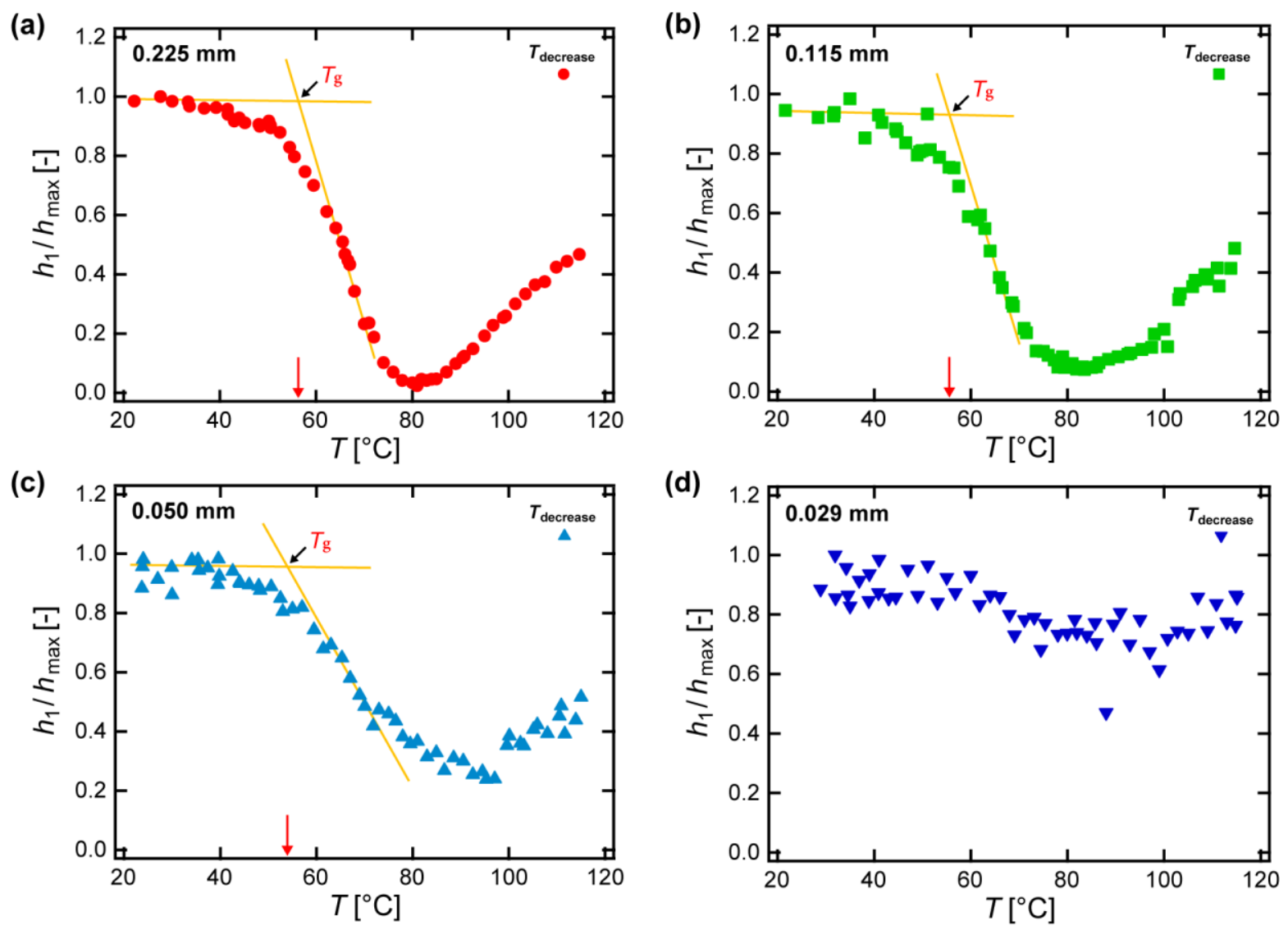

Figure S5: Results of the ball collision experiment on PLA with different film thickness. (a) film thickness $=0.225$ $\mathrm{mm} ; T_{\mathrm{g}}=56.3{ }^{\circ} \mathrm{C}$; the ratio of contact radius over film thickness $\sim 2.1$, (b) film thickness $=0.115 \mathrm{~mm} ; T_{\mathrm{g}}=55.6{ }^{\circ} \mathrm{C}$; the ratio of contact radius over film thickness $\sim 4.1$, (c) film thickness $=0.050 \mathrm{~mm}, T_{\mathrm{g}}=54.0{ }^{\circ} \mathrm{C}$; the ratio of contact radius over film thickness $\sim 9.4$, (d) film thickness $=0.029 \mathrm{~mm}$, unmeasurable $T_{\mathrm{g}}$; the ratio of contact radius over film thickness $\sim 16.2$. All the ratio values were calculated for temperature range near and under $T_{\mathrm{g}}$. 


\section{DSC results of amorphous polymers and $X_{\mathrm{c}}$ controlled polymers}
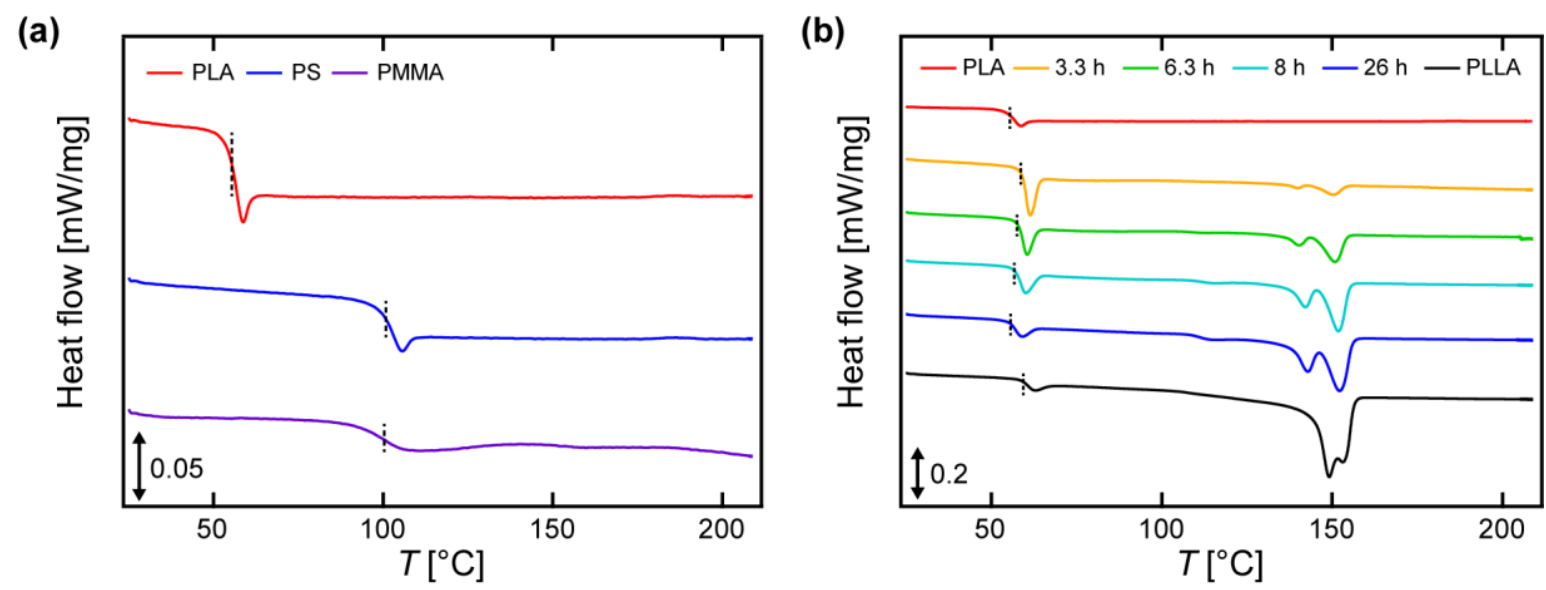

Figure S6: (a) DSC results of amorphous PLA, PS, and PMMA. Only $T_{\mathrm{g}} \mathrm{s}$ were observable. (b) DSC results of PLA, semi-crystalline PLA/PLLA 1:1 wt \% mixtures with different annealing times, and fully annealed PLLA. Except for the case of amorphous PLA, melting peaks around $150{ }^{\circ} \mathrm{C}$ are observable. $T_{\mathrm{g}} \mathrm{s}$ were determined by the inflection point of heat flow change, and are marked as black dashed lines. Relatively large enthalpy relaxation near $T_{\mathrm{g}}$ of PLA and their $1: 1 \mathrm{wt} \%$ mixtures with different annealing times was due to the long storage at room temperature before the measurement. (Heating rate: $5{ }^{\circ} \mathrm{C} / \mathrm{min}$, exo up) 


\section{DMA results of fully annealed PA6 and PET}
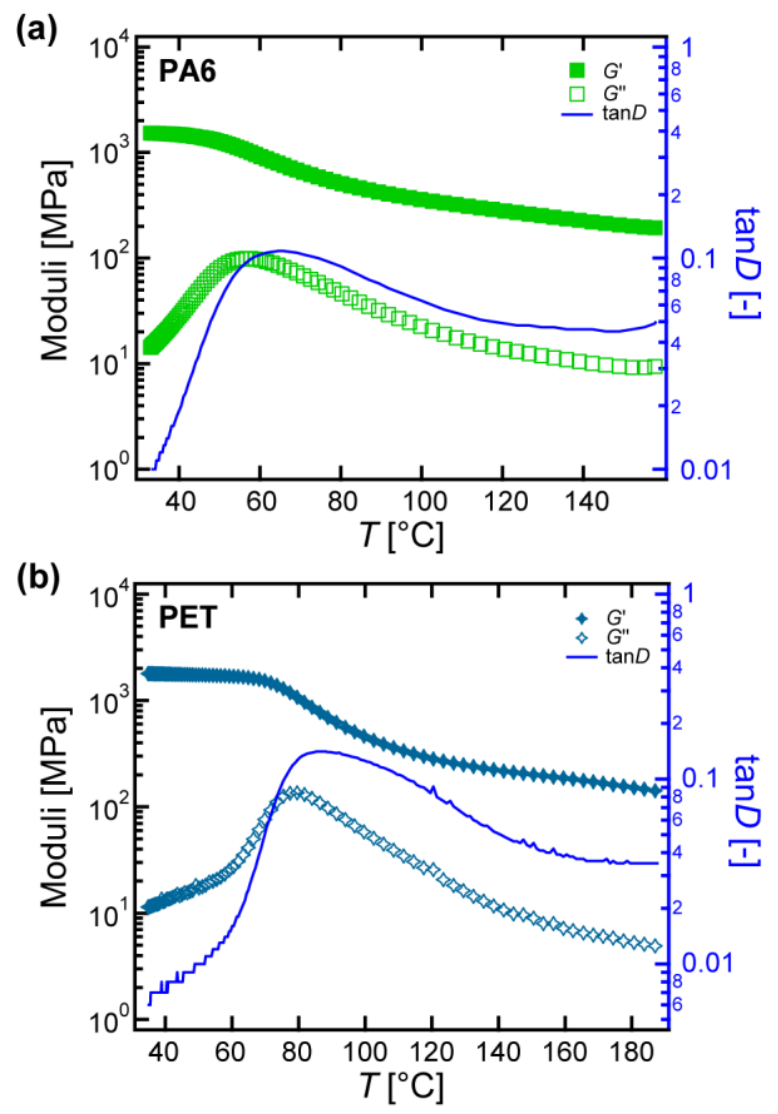

Figure S7: DMA results of (a) PA6 and (b) PET. Unlike DSC, DMA could detect $T_{\mathrm{g}}$ of semi-crystalline polymers by measuring the peak of loss modulus $\left(G^{\prime \prime}\right)$. The measurements are performed with separately prepared fully annealed polymers. (Cooling rate: $-2{ }^{\circ} \mathrm{C} / \mathrm{min}$, measuring frequency: $1 \mathrm{~Hz}$ ) 


\section{DSC result of PLA- $b$-PS}

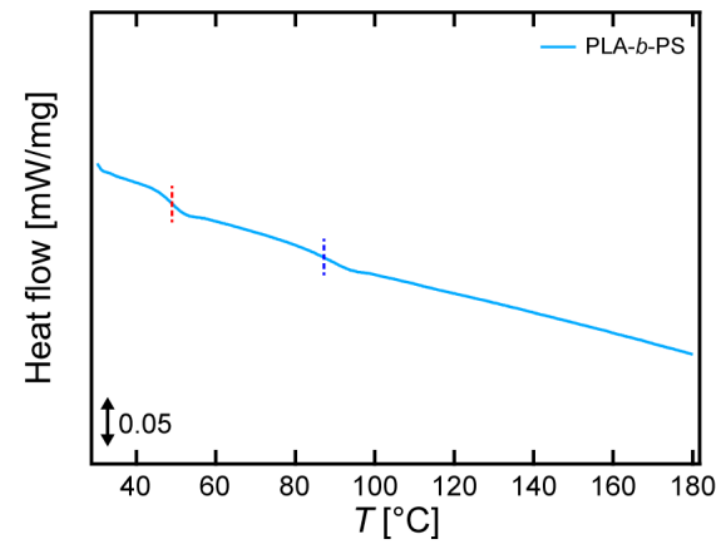

Figure S8: DSC result of PLA- $b$-PS. Two $T_{\mathrm{g}} \mathrm{s}$ were clearly observable. $T_{\mathrm{g}} \mathrm{s}$ determined by the inflection points of heat flow change are marked as a red dashed line (PLA) and a blue dashed line (PS). (Heating rate: $5{ }^{\circ} \mathrm{C} / \mathrm{min}$, exo up) 


\section{WAXD results of semi-crystalline polymers}
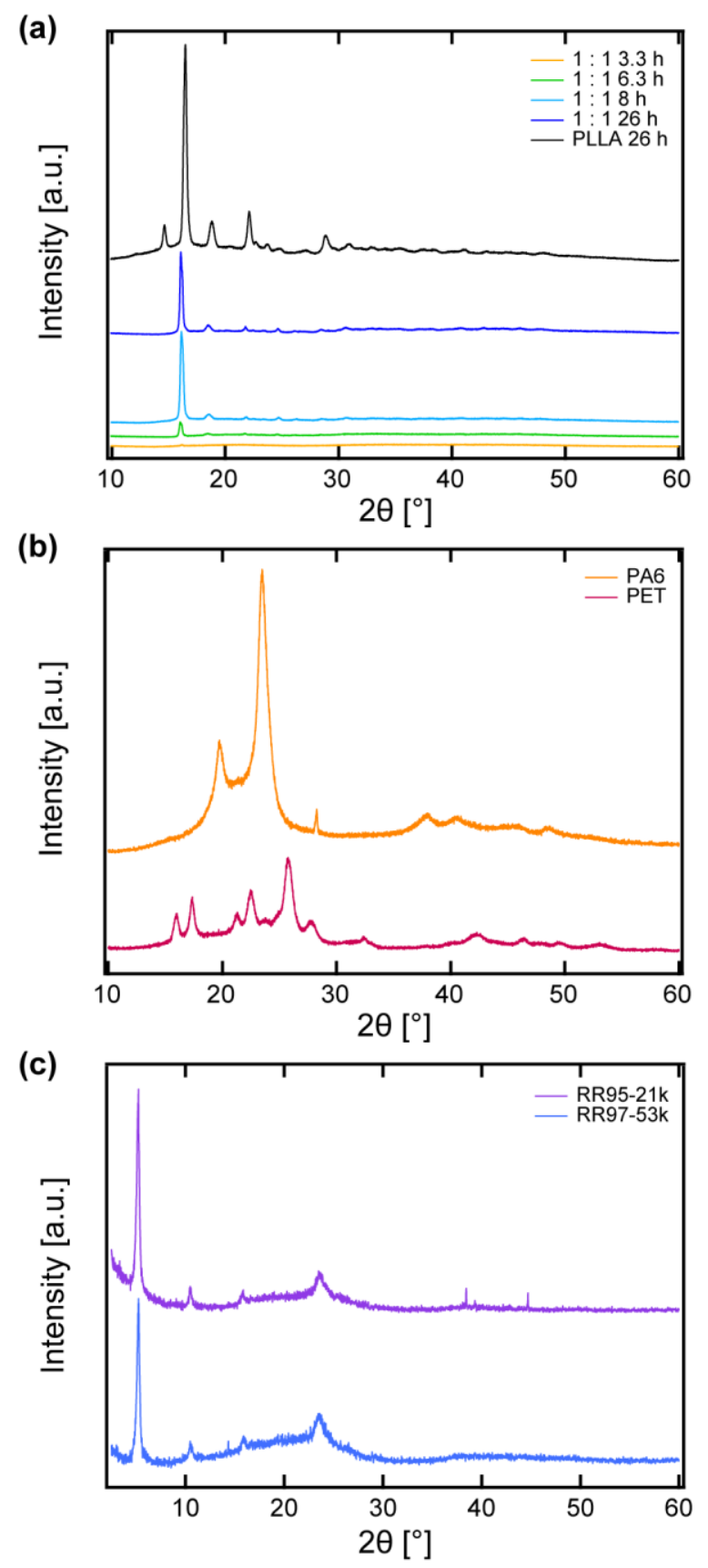

Figure S9: WAXD results of semi-crystalline polymers. (a) Fully annealed PLLA and $X_{\mathrm{c}}$ controlled polymers of PLA/PLLA 1:1 wt \% mixtures. (b) PA6 and PET. (c) Low and high $M_{\mathrm{n}}$ P3HTs. 


\section{Detailed protocols to prepare polymer films and sheets for the experiment}

PLA, PLLA, PS, and PET were pellet types, PA6 and PMMA were sheet types, PLA- $b$-PS was powder type, and P3HT was flake type. All the pellet type polymers were first rinsed with ethanol and processed to make the polymers into film or sheet form for the collision experiment. They were dried at $10^{\circ} \mathrm{C}$ below their well-known $T_{\mathrm{g}}$ under vacuum overnight, and then they were compression-molded in a $1 \mathrm{~mm}$-thick stainless steel mold at a temperature far higher than their $T_{\mathrm{g}}\left(180{ }^{\circ} \mathrm{C}\right.$ for PLA and PLLA, $210{ }^{\circ} \mathrm{C}$ for PS, and $250{ }^{\circ} \mathrm{C}$ for PET). The mold with the pellets was pressed up to 200 bar for $2 \mathrm{~min}$. To make films with different thicknesses, 0.25 and $0.12 \mathrm{~mm}$-thick aluminum molds were additionally used. For thinner films $(<0.12 \mathrm{~mm})$, the $1 \mathrm{~mm}$-thick sheet was pressed with different pressure (up to 400 bar) for 2 min without mold. Since PA6 and PMMA were sheet types, they could be directly used after rinsing and drying with the same conditions used for the pellet type polymers. All the flat sheets were then attached on the SKD-11 substrate. For the amorphous polymers, the sheets were attached on the substrate at a temperature far higher than their $T_{\mathrm{g}}$ for $15 \mathrm{~min}\left(180{ }^{\circ} \mathrm{C}\right.$ for PLA, $210{ }^{\circ} \mathrm{C}$ for PS and PMMA). For PA6 and PET, flat sheets were attached at their well-known melting temperature $\left(T_{\mathrm{m}}\right)$ and annealed at their own crystallization temperature $\left(T_{\mathrm{c}}\right)$ for at least 1 $\mathrm{h}$ to produce the maximum $X_{C}$ value before the experiment. Exceptionally, PLLA was annealed for $26 \mathrm{~h}$ due to a slow crystallization rate. ${ }^{1,2}$ For PLA- $b$-PS block copolymer, $250{ }^{\circ} \mathrm{C}$ and 100 bar condition was applied for 2 min during the compression-molding process in the $1 \mathrm{~mm}$-thick mold. The conjugated polymers (RR95-21k and RR97-53k) were compression-molded in $0.2 \mathrm{~mm}$-thick aluminum mold for $2 \mathrm{~min}$ at $280{ }^{\circ} \mathrm{C}$ and 100 bar condition. They were heated from room temperature to $280{ }^{\circ} \mathrm{C}$, compression-molded, cooled down to their own $T_{\mathrm{c}} \mathrm{s}$, annealed for $1 \mathrm{~h}$ for the crystallization, and eventually cooled down to room temperature again. The heating rate was $10{ }^{\circ} \mathrm{C} / \mathrm{min}$ and the cooling rate was $-5{ }^{\circ} \mathrm{C} / \mathrm{min}$. The final PLA- $b$-PS sheet and P3HT films were attached on the substrate with doublesided tape. Liquid nitrogen was used for lowering the temperature under room temperature.

Additionally, because of its relatively slow crystal growth rate compared to other semi-crystalline polymers, PLLA was selected as a sample for controlling $X_{\mathrm{c}}$ through different annealing times. To slow down the growth rate more for effective annealing time control and to obtain uniform spatial growth, PLA and PLLA were mixed in a 1:1 wt \% ratio in chloroform. For $1 \mathrm{~g}$ of PLA/PLLA pellets, $30 \mathrm{~g}$ of chloroform was used as a solvent and the solution was stirred at 
$300 \mathrm{rpm}$ for $4 \mathrm{~h}$ at room temperature. The mixed solution was then cast into film on a perfluoroalkoxy alkane (PFA) dish with mild drying conditions for $48 \mathrm{~h}$. After detaching from the dish, the film was dried at $45^{\circ} \mathrm{C}$ under vacuum overnight and then cut into four pieces. The flat film was then attached on SKD-11 substrate at $180{ }^{\circ} \mathrm{C}$ for 20 min. The thickness of the film after the attachment was in between 0.25 and $0.35 \mathrm{~mm}$. To control $X_{\mathrm{c}}$, the four films were separately annealed from $3.3 \mathrm{~h}$ to $26 \mathrm{~h}$ at $95{ }^{\circ} \mathrm{C}$ (about $20{ }^{\circ} \mathrm{C}$ lower than $T_{\mathrm{c}}$ ), which is close to the lower limit of crystal-forming temperature of PLLA. 


\section{Detailed protocol to determine the degree of crystallinity from WAXD data through JADE}

Step 1. Smooth the whole diffraction pattern a little using the lowest level of the Savitzky-Golay filter.

Step 2. Draw a baseline passing the first local minimum point at a small angle to the end of the measuring point (Complex polynomial function like Chebyshev polynomial is widely used for drawing the baseline.)

Step 3. Set the broad amorphous peaks (broad peak due to random diffraction). For the amorphous PLA and PET, we used a polymer sample quenched with liquid nitrogen from its melt state to determine the amorphous peaks, and for PA6, we refer to a reference. ${ }^{3}$

Step 4. Select signals from the crystalline part (sharp and narrow peaks over the broad intensity signal). These peaks are all the other peaks except the broad amorphous ones.

Step 5. As $X_{\mathrm{c}}=$ [Area of crystalline peaks / Area of total diffraction signal (amorphous part + crystalline part)], we need to find the best fit curve that matches well with the original diffraction pattern. The fit curve is the sum of every peak selected in step 3 and step 4 on the baseline. We used the pseudo-Voigt function, which is one of the best ways to fit the peaks in XRD dataset.

Step 6. Calculate $X_{\mathrm{c}}$ according to the theory explained in step 5.

Step 7. Measure the opposite side of the polymer sheet and film, and average the calculated $X_{\mathrm{c}}$ results in order to get a more accurate $X_{\mathrm{c}}$ value.

\section{Reference:}

(1) Miyata, T.; Masuko, T. Crystallization Behaviour of Poly(L-lactide). Polymer 1998, 39, 5515-5521.

(2) Androsch, R.; Di Lorenzo, M. L. Crystal Nucleation in Glassy Poly(L-lactic acid). Macromolecules 2013, 46, $6048-6056$. 
(3) Kobayashi, D.; Takahara, A. Interphase Crystal Structure of Polyamide 6 on Carbon Materials Revealed by Grazing Incidence X-Ray Diffraction with Synchrotron Radiation. Polymer 2016, 97, 174-178.

(4) Struik, L. C. E. Physical aging in amorphpus polymers and other materials; 1977. 